\title{
An Accurate and Stable Fourth Order Finite Difference Time Domain Method
}

\author{
Joshua Wilson ${ }^{1}$, Cheng Wang ${ }^{1}$, Songnan Yang ${ }^{1}$, Aly E. Fathy', and Yoon W. Kang ${ }^{2}$ \\ 1. University of Tennessee. Knoxville, TN \\ 2. Spallation Neutron Source. Oak Ridge, TN.
}

\begin{abstract}
A long-stencil fourth order finite difference method over a Yee-grid is developed to solve Maxwell's equations. The different variables are located at staggered mesh points, and a symmetric image formula is introduced near the boundary. The introduction of these symmetric ghost grid points assures the stability of the boundary extrapolation, and in turn a complete set of purely imaginary eigenvalues are given for the fourth-order discrete curl operators for both electric and magnetic fields. Subsequently, the four-stage Jameson method integrator constrained by a pre-determined time step is utilized to produce a stable full fourth order accuracy in both time and space. The accuracy of the developed numerical scheme has been validated by comparing its results to the closed form solutions for a rectangular cavity.
\end{abstract}

Index Terms - Runge-Kutta, Yee-grid, finite difference time domain, Jameson's method.

\section{INTRODUCTION}

The classical Finite Difference Time Domain (FDTD) approach introduced by Yee has been widely used in the solution of problems in electromagnetics [1]. The scheme proved to be second order accurate in space and time. However, as always in the case of a second order method, the Yee scheme begins to accumulate phase errors as time grows large, especially for electrically large domains or for late-time analysis. Because of this, several attempts have been made to extend this scheme to have a fourth order accuracy [2]. Fourth order methods allow a larger time step and courser spatial mesh to be used while maintaining the same accuracy (effectively increasing the simulation speed), or can increase the accuracy for a given mesh spacing and time step.

However, any scheme employing fourth order finite differences in space faces the problem of computing spatial derivatives near the physical boundaries. Several solutions have been proposed, such as a fourth order closure proposed in P. Petropoulous and A. Yefet [3]. An alternate approach to overcome the boundary difficulties is to introduce a compact difference operator for the spatial derivatives over the Yee stencil, as discussed in detail in J. Young [4]. Unfortunately, these methods either tend to suffer from instability near the boundary or require an implicit updating scheme.

The fourth order leapfrog or the classical fourth order Runge-Kutta method (RK4) is typically used for the fourth order time integration. The fourth order leap frog scheme leads to a corrective derivative that is very complicated to implement. The classical RK4 integrator, meanwhile, requires the storage of many temporary, intermediate field values, which increases the memory storage requirements drastically over the second order method. See J. Fang [2] and J. Young [4] for a more detailed discussion.

Recently, a fully explicit fourth order scheme over the Yee mesh grid has been proposed [5], which does not suffer from such instability near the boundary. Like Yee's original scheme, components of electric and magnetic fields are located at different (staggered) mesh points. Such a staggered grid makes the computed $\mathbf{E}$ and $\mathbf{H}$ vectors divergence-free at the discrete level.

\section{ANALYSIS SUMMARY}

To make the scheme fourth order accurate, the original FDTD method must be modified to incorporate fourth order spatial derivatives for the numerical computation of the curl. This implies that the derivatives must rely not just on the neighboring two points, but on the neighboring four points, using the appropriate four-point stencil over the MAC grid. This stencil can be expressed using the following formula [5]:

$$
\frac{d g(u)}{d u}=\frac{g\left(u-\frac{3}{2} h\right)-27 g\left(u-\frac{1}{2} h\right)+27 g\left(u+\frac{1}{2} h\right)-g\left(u+\frac{3}{2} h\right)}{24 h}
$$

Here, $\mathrm{g}$ is the appropriate field component, $\mathrm{u}$ can be any of the spatial variables $\mathrm{x}, \mathrm{y}$, or $\mathrm{z}$, while $\mathrm{h}$ is the grid spacing in the appropriate direction: either $\Delta \mathrm{x}, \Delta \mathrm{y}$, or $\Delta \mathrm{z}$. A simple Taylor expansion reveals that the error in this approximation is proportional to $h^{4}$.

While the above formula is well defined for interior mesh points, there remains the difficulty of applying it near physical boundaries, where one or more of the terms in the formula may lie outside the mesh. Instead of modifying the derivative formula for boundary points, the present scheme utilizes "ghost" points added around boundaries in such a manner as to enforce the boundary conditions and preserve the divergence free characteristic of the fields at a discrete level. An example of such a "symmetric image" formula used in this method is shown in the next section for the case of a metallic wall. This provides a completely explicit formula, and also allows for a rigorous demonstration of the stability of the method near the boundary [5].

In addition to modifying the computation of the spatial derivatives, the present method also employs a fourth order 
integration method, the Jameson method. The application of the Jameson method to the computation of electromagnetic fields was first done very recently [5], and is shown below:

$$
\begin{aligned}
& \mathbf{y}^{n,(1)}=\mathbf{y}^{n}+\frac{1}{4} \Delta t \mathbf{f}\left(y^{n}\right) \\
& \mathbf{y}^{n,(2)}=\mathbf{y}^{n}+\frac{1}{3} \Delta t \mathbf{f}\left(y^{n,(1)}\right) \\
& \mathbf{y}^{n,(3)}=\mathbf{y}^{n}+\frac{1}{2} \Delta t \mathbf{f}\left(y^{n,(2)}\right) \\
& \mathbf{y}^{n+1}=\mathbf{y}^{n}+\Delta t \mathbf{f}\left(y^{n,(3)}\right)
\end{aligned}
$$

Here, the function $\mathbf{f}$ is the numerical curl operator, and $\mathbf{y}^{n}$ is a vector containing the components of $\mathbf{E}$ and $\mathbf{H}$ at time step $n$. Such an integrator, proposed by A. Jameson in $[6,7]$ for use in the numerical simulations of gas dynamics, was only second order accurate for nonlinear gas dynamics equations. However, due to the linearity of the Maxwell equations, the Jameson method achieves fourth order accuracy for electromagnetics problems. The stability domain of the Jameson method applied to Maxwell's equations can be shown to be identical to that of the RK4 integrator, so no loss of stability occurs by using Jameson's method. The stability domain of the integrator in the complex plane is shown in Figure 1.

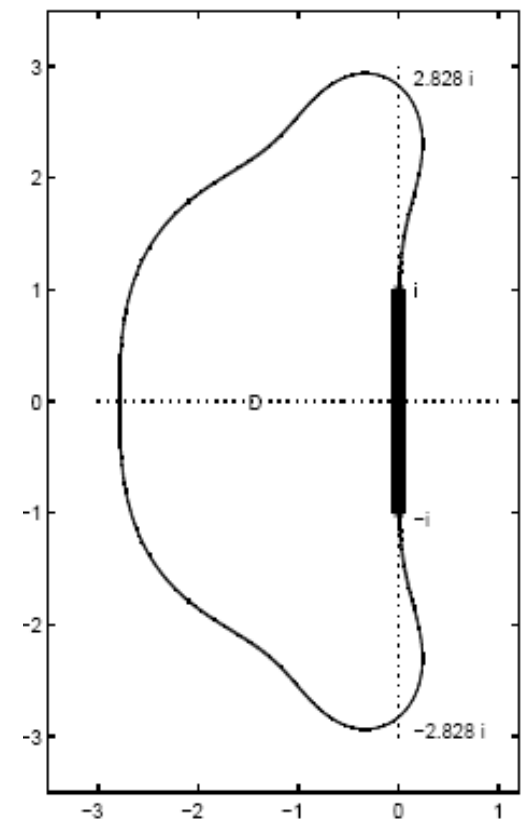

Fig. 1. Stability domain of the Jameson method fourth order time integration technique. The solid line on the imaginary axis represents that of the second order leap frog integrator.

As long as all eigenvalues of the discrete curl operator lie within the stability domain, the scheme will be stable. While the second order leapfrog integrator has a stability domain from $-\mathrm{i}$ to $+\mathrm{i}$ on the imaginary axis, the Jameson method integrator has a much larger stability domain enclosing a region in the complex plane.

It can be shown that for the case of the Maxwell equations, the Jameson method yields identical numerical results to the classical RK4 integrator [5]. However, the Jameson method is less costly to implement in one important respect. This is due to the fact that for the RK4 integrator, intermediate variables need to be saved at each intermediate stage for use in the final stage. For the Jameson method, only the result from the previous stage is needed in computing the next stage. The result is that less total memory is needed in the integration process compared to RK4.

Although the stability domain of the time integrator is improved over the classical FDTD scheme by $2 \sqrt{2}$, the eigenvalues of the fourth order discrete curl operator are larger by a factor of 7/6 [5]. Thus, the overall numeric scheme will have a CFL number of $12 \sqrt{2} / 7$ times that of the classical FDTD scheme. Therefore, the overall stability constraint for this scheme is

$$
\sqrt{\frac{1}{\mu \varepsilon}} \sqrt{\frac{1}{\Delta x^{2}}+\frac{1}{\Delta y^{2}}+\frac{1}{\Delta z^{2}}} \leq \frac{12 \sqrt{2}}{7 \Delta t} .
$$

An example of the implementation of this method over a simple rectangular domain is presented in the following section.

\section{NUMERICAL EXAMPLE}

To demonstrate this method, a simple rectangular cavity is taken under consideration. The walls are assumed to be perfect conductors. Such a cavity can support an infinite number of eigenmodes, all of which are known from basic electromagnetic theory. This makes the rectangular cavity an attractive benchmark problem. Each mode has a corresponding resonant frequency, which is also known from theory to be

$$
f_{l, m, n}=\frac{1}{2} c \sqrt{l^{2}+m^{2}+n^{2}} .
$$

where $1, \mathrm{~m}$, and $\mathrm{n}$ are non-negative integers and at least two are nonzero.

The extrapolation formulas near the boundary are very simple to implement. (Here, the treatment of the boundary conditions is demonstrated for a perfectly conducting rectangular domain, but the principles can be extended to accommodate other types of boundary conditions). The tangential electric field and normal magnetic field boundary points are computed using a Dirichlet boundary condition, while the normal electric field and tangential magnetic field boundary points are computed under a Neumann boundary 
condition. For example, at a $\mathrm{z}=$ constant boundary, the "ghost" grid points should be

$$
\begin{aligned}
& \left(E_{x}\right)_{i+1 / 2, j,-1}=-\left(E_{x}\right)_{i+1 / 2, j, 1}+O\left(h^{4}\right) \\
& \left(H_{x}\right)_{i, j+1 / 2,-1 / 2}=\left(H_{x}\right)_{i, j+1 / 2,1 / 2}+O\left(h^{5}\right) \\
& \left(H_{x}\right)_{i, j+1 / 2,-3 / 2}=\left(H_{x}\right)_{i, j+1 / 2,3 / 2}+O\left(h^{5}\right) .
\end{aligned}
$$

By differentiating the original wave equation, this extrapolation technique is shown to be fourth order accurate[5].

Three schemes were selected for comparison with this benchmark problem. The first is the original FDTD, which is second order in time and space (or 2X2). The second is a scheme employing a second order leapfrog integrator in time and a fourth order finite difference stencil in space (2X4). The third is the newly proposed $4 \mathrm{X} 4$ scheme.

The dimensions of the cavity were chosen to be $1 \mathrm{~m}$ by $1 \mathrm{~m}$ by $1 \mathrm{~m}$. The initial condition was chosen to force the initial magnetic field to be zero, and the initial electric field to be a sum over many cavity eigenmodes, giving the appearance of a "random" initial condition. The amplitude of the $1, \mathrm{~m}, \mathrm{n}$ eigenmode in the initial condition was given by

$$
A_{l, m, n}=\frac{1}{l^{2}+m^{2}+n^{2}},
$$

where $1, \mathrm{~m}$, and $\mathrm{n}$ all range from 1 to 20 , for a total of 8000 modes. However, due to the decaying amplitude for large $\mathrm{m}$, $\mathrm{n}$, and $\mathrm{l}$, only a few of these modes will be significant in the spectral distribution.

This choice of initial condition guarantees that $\mathrm{E}$ and $\mathrm{H}$ are divergence free and satisfy the boundary conditions at the cavity walls.

\section{RESULTS}

Running the 4X4 scheme and taking an FFT of the electric field component Ey at a sample observation point, the spectrum of Figure 2 was obtained.

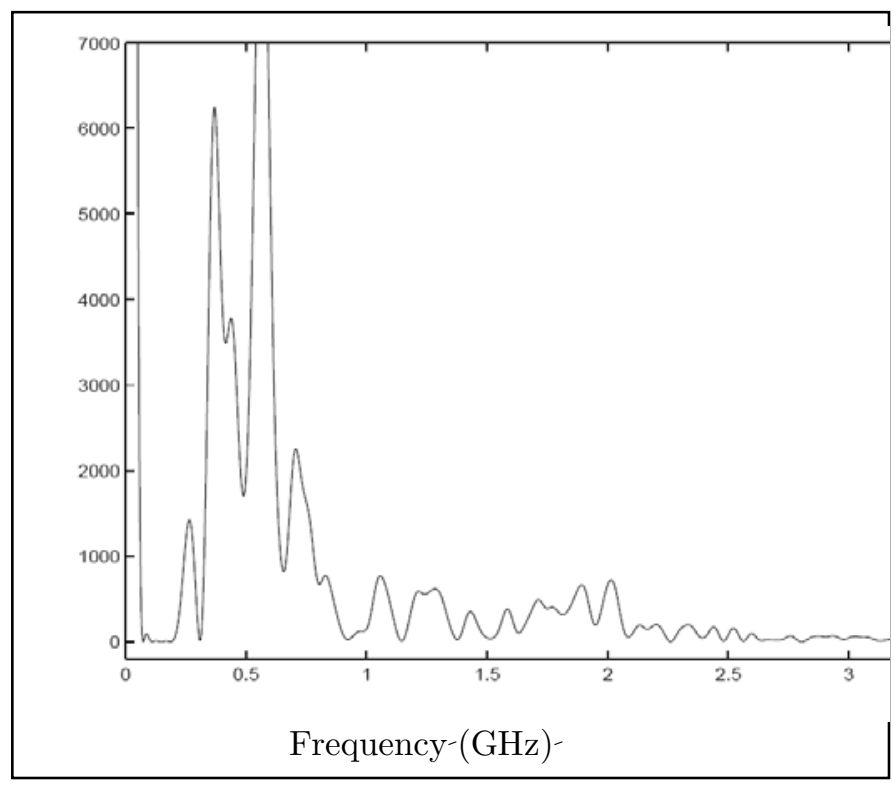

Fig. 2. Spectrum of Ey at a sample observation point. A multimode initial condition was purposely chosen.

For the trial simulation, the TE333 mode, where $1=3, \mathrm{~m}=$ 3 , and $n=3$, was chosen to be measured. This mode is a good test of the fourth order scheme because it has rapidly varying fields in the $\mathrm{x}, \mathrm{y}$, and $\mathrm{z}$ directions. The theoretical resonant frequency for this mode is $778.88 \mathrm{MHz}$.

The accuracy of the simulations was measured by taking a dot product of the cavity fields with the known modal distribution of the 3,3,3 mode at each time step, in an effort to select only the mode of interest. An FFT is then performed with an appropriate window function, in this case a raised cosine. Then, the frequency of the peak in the Fourier power spectrum is compared to the known resonant frequency.

A number of simulations were performed for each scheme, with different numbers of grid points $\mathrm{N}$, hence varying $\Delta x$ (with the assumption $\Delta x=\Delta y=\Delta z$ ). $\frac{\Delta t}{\Delta x}$ was fixed by the most restrictive CFL condition (the $2 \mathrm{X} 4$ case). The final time was also fixed.

The error of the $2 \times 2,2 \times 4$, and $4 \times 4$ schemes, regarding the frequency of the mode $1=3, m=3, n=3$ mode, is shown in Figure 3. 


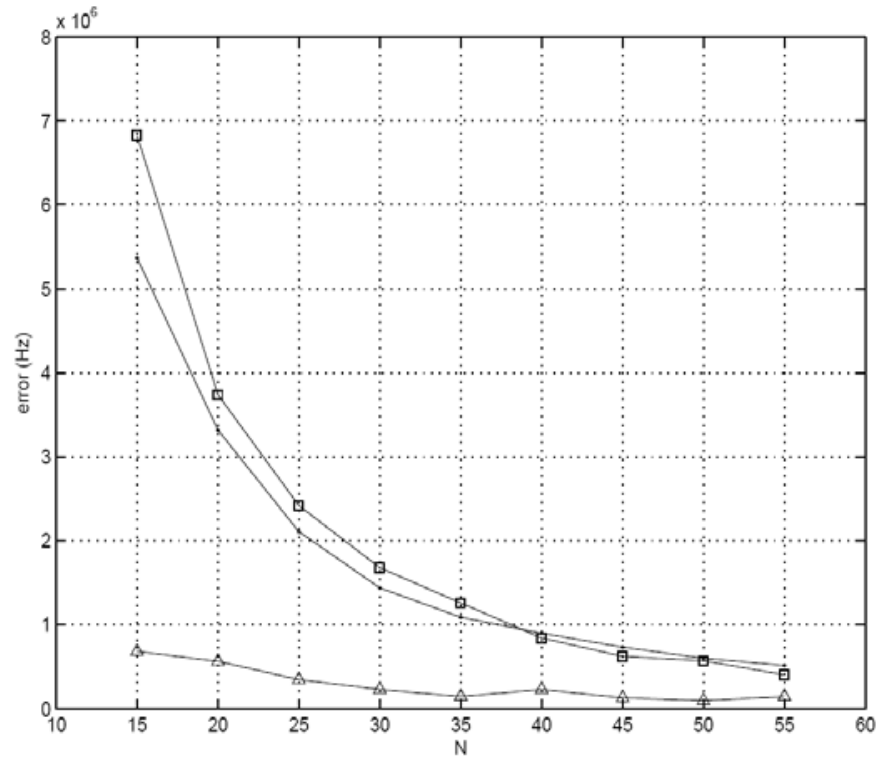

Fig. 3. Error comparison between the $2 \mathrm{X} 2,2 \mathrm{X} 4$, and proposed $4 \mathrm{X} 4$ method for the TE333 mode. The dot line represents the $2 \mathrm{X} 2$ scheme; the square line, the $2 \mathrm{X} 4$ scheme, and the triangle line, the $4 \mathrm{X} 4$ scheme.

It is obvious that the proposed fourth order method provides much more accurate simulation results than those of the $2 \times 2$ and 2 X 4 methods. It is also interesting to note that there is relatively little difference between the $2 \times 2$ method and the $2 \mathrm{X} 4$ method. This indicates that moving to a fourth order stencil in space is not significantly advantageous if a second order time integration is still employed. Only in the case of a fully fourth order scheme in space and time can a significant advantage be noticed.

Such an accuracy can even be observed for a relatively course mesh. For a 20 X 20 X 20 grid, for example, an error of $632 \mathrm{kHz}$ is observed. By comparison, a 50 X 50 X 50 grid in CST, a commercial electromagnetic simulation software, gives an error of $1.15 \mathrm{MHz}$.

\section{CONCLUSION}

The resulting scheme is shown to preserve fourth order accuracy in space and time for the linear Maxwell equations. Moreover, the symmetric image formula yields a completely explicit and stable updating scheme. Due to the Jameson method integration, the method also saves memory over schemes which employ a more conventional RK4 integrator. At the same time, it offers a larger stability domain than the second order scheme. The overall fourth order scheme has a maximum CFL number of $12 \sqrt{2} / 7$ times that of the original FDTD algorithm, which is a drastic improvement. This implies that a larger time step can be used in the simulation without sacrificing stability. This property makes the present method attractive for electrically large domains or late-time analysis.

\section{ACKNOWLEDGEMENT}

This work has been sponsored by ORNL-SNS. The Spallation Neutron Source is managed by UT-Battelle, LLC, under contract DE-AC05-00OR22725 for the U.S. Department of Energy.

\section{REFERENCES}

[1] K.S. Yee, "Numerical solution of initial boundary value problems involving Maxwell's equations in isotropic media," IEEE Trans. Antennas Propagate. Vol. 14, pp. 302-307, 1966. [2] J. Fang, "Time domain Finite Difference Computation for Maxwell equations", Ph.D dissertation, Univ. California, Berkeley, 1989.

[3] P. G. Petropoulous and A. Yefet, "Subgridding a fourth order FDTD scheme for Maxwell's equations," Proceedings of Fourth International Workshop on Computational Electromagnetics in the Time-Domain: TLM/FDTD and Related Techniques (CEM-TD). pp. 39-45, 2001.

[4] J. Young, D. Gaitonde and J. Shang, "Toward the construction of a fourth-order difference scheme for transient EM wave simulation: staggered grid approach," IEEE Trans. Antennas Propagate. Vol. 45, pp. 1573-1580, 1997

[5] Joshua Wilson, Cheng Wang, Aly Fathy, Yoon Kang. "Development of a Fourth Order FDTD Algorithm Implementing Symmetric Boundary Extrapolation and Jameson Method Time Stepping". Submitted for publication in IEEE Transactions on Microwave Theory and Techniques. Feb. 2008.

[6] A. Jameson and P. D. Lax, "Conditions for the construction of multi-point total variation diminishing difference schemes", Appl. Numer. Math. Vol. 2. pp. 335-345, 1986.

[7] A. Jameson, W. Schmidt and E. Turkel, "Numerical solution of the Euler equations by finite volume methods using Runge-Kutta time stepping schemes", AI AA Paper. pp. 81-1259, 1981. 